SSN 2519-7398 (Versión electrónica)

DOI: http://dx.doi.org/10.21704/ac.v78i2.1060

(C) Universidad Nacional Agraria La Molina, Lima - Perú

\title{
Riego y uso de antitranspirantes sobre la vida postcosecha de Heliconia bihai L.
}

\author{
Irrigation and antitranspirants use on Heliconia bihai L. postharvest life
}

\author{
Andrés Alcalá B. ${ }^{1}$; José Palacios V. ${ }^{2}$ \\ *Autor de correspondencia
}

\begin{abstract}
Resumen
En el cultivo de Heliconia bihai L., se evaluó el efecto de la aplicación de riego antes de la cosecha de sus inflorescencias y la aplicación de los antitranspirantes comerciales Stress Relief 35® y Vapor Gard ${ }^{\circledR}$, a diferentes dosis $(0,0.5$ y $1 \%)$, antes o después de su cosecha. Los resultados fueron medidos a través de la observación poscosecha del porcentaje de pérdida de peso, el porcentaje de daño observado, los límites de su comercialización y vida en florero. La aplicación de riego antes de la cosecha en combinación con la aplicación de Vapor Gard® antes o después de la cosecha produjo una menor pérdida de peso. La aplicación de riego antes de la cosecha, en combinación con la aplicación de Vapor Gard® al 1\%, redujo el porcentaje de daño observado. La aplicación de riego en combinación con algún antitranspirante en pre o poscosecha, prolongó su vida en florero. La aplicación del antitranspirante Vapor Gard® al 1\%, produjo menor pérdida de peso, menor daño observado, mayor límite de comercialización y mayor vida en florero. Finalmente la aplicación de riego antes de la cosecha en cualquiera de los antitranspirantes estudiados en dosis del 1\% o cualquiera de los antitranspirantes aplicados luego de la cosecha, redujeron la pérdida de peso y el daño observado, prolongando el límite de comercialización y vida en florero de las inflorescencias de Heliconia bihai.
\end{abstract}

Palabras clave: Heliconia bihai; poscosecha; riego; antitranspirantes.

\begin{abstract}
In cultivated Heliconia bihai L., the application of irrigation water before harvesting inflorescences and applying commercial antitranspirants Stress Relief $35 \AA$ and Vapor Gard ${ }^{\circledR}$ at different doses $(0,0.5$ and $1 \%)$, before or after harvest, was evaluated. The results were measured by viewing postharvest weight loss percentage, percentage of observed damage, the limits of their marketing and vase life. Irrigation application before harvesting in combination with Vapor Gard ${ }^{\circledR}$ before or after harvest was a reduced weight loss. Application of irrigation prior to harvest, combined with the application of Vapor Gard ® 1\%, reduced the percentage of damage observed. The application of irrigation water in combination with any pre or post antitraspirant, extended vase life. Application of Vapor Gard ${ }^{\circledR} 1 \%$, produced less weight loss, less damage observed, higher marketing limits and increased vase life. Finally, the application of irrigation water prior to harvest, with any of the antitranspirants studied at doses of $1 \%$ or antitranspirants applied after harvest, reduced weight loss and the observed damage, extending the limit marketing and vase life of inflorescences of Heliconia bihai.
\end{abstract}

Key words: Heliconia bihai; postharvest; irrigation; antitranspirants.

\section{Introducción}

El cultivo de flores tropicales en la región ha surgido como una alternativa de producción y desarrollo para los agricultores del Perú y de Sudamérica debido a un significativo incremento porcentual de la demanda de este tipo de flores, cuya participación tanto en el mercado nacional como internacional ha crecido notablemente en los últimos años (Rocha, 2010).De esta manera, diversas especies del género Heliconia exhiben hoy un gran potencial para la producción de flor cortada (Torres, 2003). Dentro de este género, la Heliconia bihai es una especie de importancia comercial, tanto por su bella inflorescencia, firme y erecta, fácil de empacar y transportar, como por su aceptable vida poscosecha (Castro et al., 2006). A pesar de esto, su introducción en los mercados no ha sido tan rápida como se esperaba, debido a que se han encontrado algunos problemas en su producción y comercialización, entre los que se incluyen: tamaño y peso del producto, temperaturas de almacenamiento y procedimientos de manejo poscosecha. Además, su producción es limitada y se ve particularmente afectada por la falta de información sobre su manejo agronómico (Rocha, 2010). Por eso es de gran importancia 
ofrecer alternativas prácticas en el manejo poscosecha y obtener métodos que preserven la calidad de estas flores y permitan una mejor competencia en el mercado. Este estudio pretende aportar conocimientos fundamentales para el manejo poscosecha de inflorescencias de Heliconia bihai empleando tratamientos que prolonguen su vida y conserven la calidad poscosecha, mediante la hidratación de las inflorescencias a través de un riego previo a la cosecha y la reducción de la transpiración mediante el uso de antitranspirantes.

\section{Materiales y métodos}

Se evaluó el efecto de la aplicación o no de riego 12 horas antes de la cosecha y la aplicación de los antitranspirantes Stress Relief $35 \AA$ y Vapor Gard ${ }^{\circledR}$ a dosis de $0,0.5$ y 1 por ciento aplicados 24 horas antes de la cosecha o inmediatamente después de la misma. Las evaluaciones fueron hechas sobre el porcentaje de pérdida de peso, el porcentaje de daño observado, los días de límite de comercialización y los días de vida en florero de inflorescencias de Heliconia bihai.

\section{Pérdida de peso}

Se determinó diariamente, el porcentaje de pérdida de peso de las inflorescencias, desde el primer día de cosecha hasta 18 días después.

\section{Daño observado}

Se determinó diariamente el porcentaje de daño observado sobre la superficie de las brácteas cincinales (deshidratación, amarillamiento, pardeamiento o manchas necróticas) desde el primer día de la cosecha hasta 18 días después.

\section{Límite de comercialización}

El límite de comercialización en las inflorescencias de Heliconia bihai se determinó cuando el daño observado sobre la superficie de las brácteas cincinales ocupó más del 20 por ciento de su superficie total. El valor del límite de comercialización fue determinado por el número de días máximo en los que las inflorescencias podían ser comercializadas por los agentes minoristas (florerías), de tal manera que aún quedaran algunos días para el uso del consumidor final.

\section{Vida en florero}

La vida en florero de las inflorescencias de Heliconia bihai se determinó cuando el daño observado, sobre la superficie de las brácteas cincinales, ocupó más del 40 por ciento de su superficie. El valor de la vida en florero fue determinado por el número de días máximo en los que las inflorescencias podían ser utilizadas por el consumidor final, luego de lo cual fueron descartadas. Las operaciones realizadas en el presente experimento se detallan a continuación:

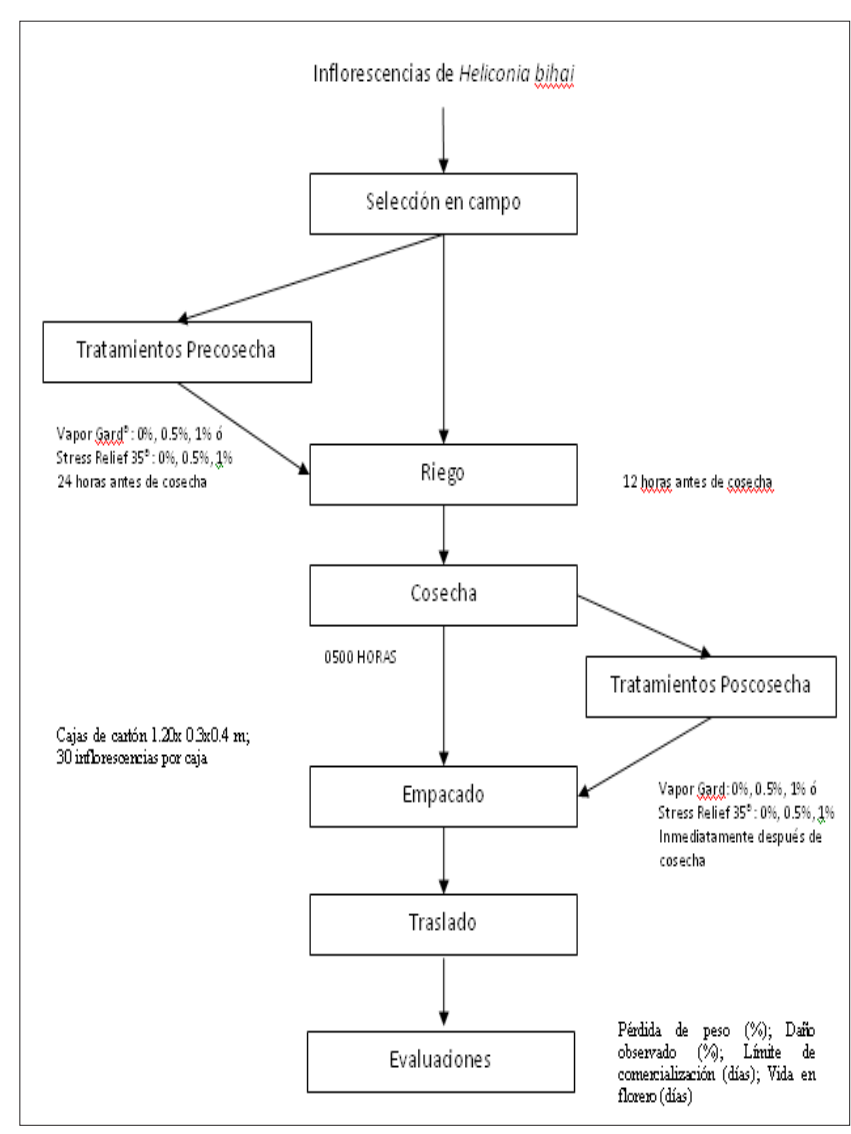

Figura 1. Esquema de las operaciones realizadas en el experimento

\section{Resultados y discusión}

\section{Pérdida de peso}

El ANVA indicó que en dos interacciones: (1) aplicación de riego, tipo de antitranspirante y momento de aplicación y (2) tipo y dosis de antitranspirante; así como en los factores simples estudiados (aplicación de riego; tipo, momento de aplicación y dosis de antitranspirantes), tuvieron un efecto estadísticamente significativo sobre la pérdida de peso (\%) con un 95.0 por ciento de nivel de confianza ( $p$-valor $<0.05$ ). En la primera interacción (riego, tipo de antitranspirante y momento de aplicación), las inflorescencias de plantas que recibieron riego y antitranspirante Vapor Gard $\AA$ en pre y poscosecha mostraron una pérdida de peso significativamente menor, $31.8 \%$ y $31.9 \%$ respectivamente, que el resto de tratamientos (Tabla 1). En la segunda interacción, tipo de antitranspirante y dosis, aquellas inflorescencias que recibieron el antitranspirante Vapor Gard $\AA$ al $1 \%$ mostraron una pérdida de peso significativamente menor $30.8 \%$ que aquellas que recibieron en antitranspirante Stress Relief $35 \AA$, en cualquiera de las dosis, y el testigo (Tabla 2). En el factor simple: riego, las inflorescencias de plantas que fueron regadas antes de la cosecha mostraron una pérdida de peso significativamente menor (33.2 por ciento) que aquellas que no lo recibieron, 35.4 por ciento (Tabla 3). 
En el factor simple: tipo de antitranspirante, Vapor Gard $\mathbb{R}$ o Stress Relief $35 \AA$, las inflorescencias que fueron tratadas con Vapor Gard $\AA$ tuvieron una pérdida de peso significativamente menor, 33.2 por ciento, que las tratadas con Stress Relief $35 \AA$, 35.1 por ciento (Tabla 3 ).

Tabla 1. Pérdida de peso en la interacción: aplicación de riego, tipo y momento de aplicación del antitranspirante

\begin{tabular}{|c|c|c|c|}
\hline \multirow[b]{2}{*}{ Riego } & \multicolumn{2}{|c|}{ Interacción } & \multirow{2}{*}{$\begin{array}{l}\text { Pérdida } \\
\text { de peso }\end{array}$} \\
\hline & Antitranspirante & Momento de aplicación & \\
\hline \multirow{4}{*}{ Sin riego } & Stress Relief $35 \AA$ & precosecha & $36.0 \mathrm{bc}$ \\
\hline & Stress Relief $35 \AA$ & poscosecha & $36.4 \mathrm{c}$ \\
\hline & Vapor Gard $®$ & precosecha & $35.7 \mathrm{bc}$ \\
\hline & Vapor Gard $\AA$ & poscosecha & $33.5 \mathrm{bc}$ \\
\hline \multirow{4}{*}{ Con riego } & Stress Relief $35 \circledR$ & precosecha & $34.9 \mathrm{bc}$ \\
\hline & Stress Relief $35 \AA$ & poscosecha & $32.9 \mathrm{~b}$ \\
\hline & Vapor Gard $®$ & precosecha & $31.8 \mathrm{a}$ \\
\hline & Vapor Gard $\AA$ & poscosecha & $31.9 \mathrm{a}$ \\
\hline
\end{tabular}

Tabla 2. Pérdida de peso en la interacción: tipo y dosis de antitranspirante

\begin{tabular}{|c|c|c|}
\hline \multicolumn{2}{|c|}{ Interacción } & \multirow{2}{*}{ Pérdida de peso (\%) } \\
\hline Antitranspirante & Dosis $(\%)$ & \\
\hline \multirow{3}{*}{ Stress Relief $35 \circledR$} & 0.0 & $36.9 \mathrm{c}$ \\
\hline & 0.5 & $34.2 \mathrm{~b}$ \\
\hline & 1.0 & $34.1 \mathrm{~b}$ \\
\hline \multirow{3}{*}{ Vapor Gard $\mathbb{R}$} & 0.0 & $36.9 \mathrm{c}$ \\
\hline & 0.5 & $31.9 \mathrm{ab}$ \\
\hline & 1.0 & $30.8 \mathrm{a}$ \\
\hline
\end{tabular}

Tabla 3. Pérdida de peso en los factores simples: aplicación de riego, tipo de antitranspirante, dosis y momento de cosecha

\begin{tabular}{|c|c|c|c|c|c|c|c|}
\hline Riego & \multicolumn{2}{|c|}{ Antitranspirante } & \multicolumn{3}{|c|}{ Dosis (\%) } & \multicolumn{2}{|c|}{ Cosecha } \\
\hline Sin Con & $\begin{array}{l}\text { Stress } \\
\text { Relief }\end{array}$ & $\begin{array}{l}\text { Vapor } \\
\text { Gard }\end{array}$ & 0.0 & 0.5 & 1.0 & Pre & Post \\
\hline $4 \mathrm{~b} 33.2 \mathrm{a}$ & $35.1 \mathrm{~b}$ & $33.2 \mathrm{a}$ & 36.9 & & 32.4 & & 0.0 \\
\hline
\end{tabular}

En el factor simple: dosis de aplicación de antitranspirante, las inflorescencias que recibieron la dosis más alta, 1 por ciento, fueron las que mostraron una pérdida de peso significativamente menor, 32.4 por ciento, que las demás concentraciones, 0 por ciento y 0.5 por ciento, 36.9 por ciento y 34.6 por ciento respectivamente (Tabla 3). En el factor simple: momento de aplicación del antitranspirante, las inflorescencias tratadas con antitraspirantes luego de ser cosechadas, tuvieron una pérdida de peso significativamente menor, 33.6 por ciento, que aquellas inflorescencias tratadas antes de ser cosechadas (Tabla 3).

\section{Daño observado}

El ANVA indicó que en dos interacciones:(1) aplicación de riego, tipo y dosis de antitranspirante y (2) tipo y dosis de antitranspirante; así como los factores simples estudiados (aplicación de riego; tipo, momento de aplicación y dosis de antitranspirantes, tuvieron un efecto estadísticamente significativo sobre el daño observado (\%) con un 95.0 por ciento de nivel de confianza ( $\mathrm{p}$-valor $<0.05$ ). En la primera interacción, riego, tipo de antitranspirante y dosis del mismo, las inflorescencias de plantas que recibieron riego y antitranspirante Vapor Gard $\AA$ al 1 por ciento, mostraron un promedio significativamente menor de daño, 37.5 por ciento, que el resto de inflorescencias (Tabla 4).

Tabla 4. Daño observado en la interacción: tipo y dosis de antitranspirante

\begin{tabular}{|c|c|c|}
\hline \multicolumn{2}{|c|}{ Interacción } & \multirow{2}{*}{ Daño observado (\%) } \\
\hline Antitranspirante & Dosis & \\
\hline \multirow{3}{*}{ Stress Relief $35 \AA$} & 0.0 & $49.0 \mathrm{~b}$ \\
\hline & 0.5 & $47.8 \mathrm{~b}$ \\
\hline & 1.0 & $46.0 \mathrm{~b}$ \\
\hline \multirow{3}{*}{ Vapor Gard® } & 0.0 & $49.0 \mathrm{~b}$ \\
\hline & 0.5 & $46.0 \mathrm{~b}$ \\
\hline & 1.0 & $41.5 \mathrm{a}$ \\
\hline
\end{tabular}

En la segunda interacción (tipo de antitranspirante y dosis del mismo), los resultados muestran que aquellas inflorescencias que recibieron el antitranspirante Vapor Gard $\AA$ al 1\% mostraron un promedio significativamente menor de daño en sus inflorescencias (41.5\%) que aquellas que fueron tratadas con Stress Relief $35 \AA$ en cualquiera de sus dosis y que Vapor Gard $\AA$ en sus dosis más bajas (Tabla 5).

Tabla 5. Daño observado en la interacción: riego, tipo y dosis del antitranspirante

\begin{tabular}{|c|c|c|c|}
\hline \multicolumn{3}{|c|}{ Interacción } & \multirow{2}{*}{$\begin{array}{c}\text { Daño observado } \\
(\%)\end{array}$} \\
\hline Riego & Antitranspirante & Dosis & \\
\hline \multirow{6}{*}{ Sin riego } & Stress Relief 35® & 0.0 & $50.0 \mathrm{c}$ \\
\hline & Stress Relief 35® & 0.5 & $50.0 \mathrm{c}$ \\
\hline & Stress Relief $35 \AA$ & 1.0 & $47.5 \mathrm{bc}$ \\
\hline & Vapor Gard® & 0.0 & $50.0 \mathrm{c}$ \\
\hline & Vapor Gard $\AA$ & 0.5 & $47.0 \mathrm{bc}$ \\
\hline & Vapor Gard® & 1.0 & $45.5 \mathrm{bc}$ \\
\hline \multirow{6}{*}{ Con riego } & Stress Relief 35® & 0.0 & $48.0 \mathrm{bc}$ \\
\hline & Stress Relief 35® & 0.5 & $45.5 \mathrm{bc}$ \\
\hline & Stress Relief 35® & 1.0 & $44.5 \mathrm{~b}$ \\
\hline & Vapor Gard® & 0.0 & $48.0 \mathrm{bc}$ \\
\hline & 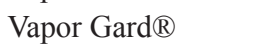 & 0.5 & $45.0 \mathrm{~b}$ \\
\hline & Vapor Gard ( & 1.0 & $37.5 \mathrm{a}$ \\
\hline
\end{tabular}

En el factor aplicación de riego, las inflorescencias de plantas que recibieron riego antes de ser cosechadas, mostraron un daño significativamente menor $44.8 \%$ que aquellas que no fueron regadas antes de ser cosechadas, $48.3 \%$ (Tabla 6). En el factor simple: tipo de antitranspirante, Vapor Gard $\AA$ o Stress Relief $35 \AA$, las inflorescencias que fueron tratadas con Vapor Gard $\AA$ mostraron un daño significativamente menor 45.5\% que las tratadas con Stress Relief 35®, $47.6 \%$ (Tabla 6 )

En el factor simple: dosis de antitranspirante, las 
inflorescencias que recibieron dosis de 0.5 por ciento y 1 por ciento tuvieron resultados significativamente menores que las que no recibieron antitranspirante, 49 por ciento. Sin embargo aquellas inflorescencias que recibieron antitranspirante a dosis del 1 por ciento mostraron daños significativamente menores, 43.8 por ciento, que a las inflorescencias tratadas a dosis del 0.5 por ciento, 46.9 por ciento (Tabla 6). En el factor simple: momento de aplicación del antitranspirante, las inflorescencias tratadas con antitraspirantes después de ser cosechadas, tuvieron un daño significativamente menor $45.7 \%$ que aquellas inflorescencias tratadas antes de ser cosechadas $47.4 \%$ (Tabla 6).

Tabla 6. Daño observado en los factores simples: riego, tipo de antitranspirante dosis y momento de cosecha

\begin{tabular}{|c|c|c|c|c|c|c|c|c|}
\hline \multicolumn{2}{|c|}{ Riego } & \multicolumn{2}{|c|}{ Antitranspirante } & \multicolumn{3}{|c|}{ Dosis $(\%)$} & \multicolumn{2}{|c|}{ Cosecha } \\
\hline Sin & Con & $\begin{array}{l}\text { Stress } \\
\text { Relief }\end{array}$ & $\begin{array}{l}\text { Vapor } \\
\text { Gard }\end{array}$ & 0.0 & 0.5 & 1.0 & Pre & Post \\
\hline 8.3 & & $47.6 \mathrm{~b}$ & $45.5 \mathrm{a}$ & & & & & $45.7 \mathrm{a}$ \\
\hline
\end{tabular}

\section{Límite de comercialización}

El ANVA indicó que en una interacción: (1) entre el tipo y dosis de antitranspirante; así como los factores estudiados, aplicación de riego; tipo, momento de aplicación y dosis de antitranspirantes, tuvieron un efecto estadísticamente significativo sobre límite de comercialización (días) con un 95.0 por ciento de nivel de confianza ( $p$-valor $<0.05$ ).

En la interacción, tipo de antitranspirante y dosis del mismo, los resultados muestran que aquellas plantas que recibieron el antitranspirante Vapor Gard ${ }^{\circledR}$ al 1 por ciento y 0.5 por ciento mostraron un límite de comercialización significativamente mayor, 13.5 días y 12.3 días respectivamente. Por otro lado las inflorescencias a las que se aplicó Stress Relief $35 \AA$ en dosis de 1 por ciento (11.3 días) y 0.5 por ciento ( 10.8 días) mostraron diferencias significativas con las inflorescencias cuya dosis de antitranspirante aplicado fue de 0 por ciento, 9.7 días (Tabla 7).

Tabla 7. Límite de comercialización en la interacción: tipo $\mathrm{y}$ dosis de antitranspirante

\begin{tabular}{|c|c|c|}
\hline \multicolumn{2}{|c|}{ Interacción } & \multirow{2}{*}{$\begin{array}{l}\text { Límite de Comercialización } \\
\text { (días) }\end{array}$} \\
\hline Antitranspirante & Dosis & \\
\hline \multirow{3}{*}{ Stress Relief $35 \AA$} & 0.0 & $9.7 \mathrm{a}$ \\
\hline & 0.5 & $10.8 \mathrm{~b}$ \\
\hline & 1.0 & $11.3 \mathrm{bc}$ \\
\hline \multirow{3}{*}{ Vapor Gard $®$} & 0.0 & $9.7 \mathrm{a}$ \\
\hline & 0.5 & $12.3 \mathrm{~cd}$ \\
\hline & 1.0 & $13.5 \mathrm{~d}$ \\
\hline
\end{tabular}

En el factor simple: aplicación de riego, las inflorescencias de plantas que recibieron riego antes de ser cosechadas, tuvieron un límite de comercialización significativamente mayor (12.4 días) que aquellas que no recibieron riego antes de ser cosechadas, 10.1 días (Tabla 8).
Tabla 8. Límite de comercialización en los factores simples: aplicación de riego, tipo de antitranspirante, dosis y momento de cosecha

\begin{tabular}{cccccccc}
\hline \multicolumn{2}{c}{ Riego } & \multicolumn{2}{c}{ Antitranspirante } & \multicolumn{2}{c}{ Dosis (\%) } & \multicolumn{2}{c}{ Cosecha } \\
\hline Sin Con & $\begin{array}{c}\text { Stress } \\
\text { Relief }\end{array}$ & $\begin{array}{c}\text { Vapor } \\
\text { Gard }\end{array}$ & 0 & 0.5 & 1 & Pre & Post \\
& $10.1 \mathrm{a} 12.4 \mathrm{~b}$ & $10.6 \mathrm{a}$ & $11.8 \mathrm{~b}$ & $9.7 \mathrm{a}$ & $11.6 \mathrm{~b}$ & $12.4 \mathrm{c}$ & $10.9 \mathrm{a} 11.5 \mathrm{~b}$ \\
\hline
\end{tabular}

En el factor simple: tipo de antitranspirante (Vapor Gard ${ }^{\circledR}$ o Stress Relief 35®), las inflorescencias que fueron tratadas con Vapor Gard ${ }^{\circledR}$ tuvieron un límite de comercialización significativamente mayor (11.8 días) que las tratadas con Stress Relief $35 \AA$, 10.6 días (Tabla 8 ).

En el factor simple: dosis de aplicación de antitranspirante, las inflorescencias que recibieron las dosis más altas (1 por ciento) fueron las que tuvieron resultados significativamente superiores (12.4 días) a las demás concentraciones (0.5 por ciento y 0 por ciento). Cabe resaltar, que aquellas inflorescencias que recibieron antitranspirante a una dosis de 0.5 por ciento también tuvieron un límite de comercialización significativamente mayor (11.6 días) que las inflorescencias que no recibieron antitranspirante, 9.7 días (Tabla 8).

En el factor simple: momento de aplicación del antitranspirante, las inflorescencias tratadas con después de ser cosechadas, tuvieron un límite de comercialización significativamente mayor (11.5 días) que aquellas inflorescencias tratadas antes de ser cosechadas (10.9 días) (Tabla 8).

\section{Vida en florero}

El ANVA indicó que en dos interacciones: (1) entre la aplicación de riego y el momento de aplicación del antitranspirante y (2) entre el tipo y dosis de antitranspirante; así como en los factores simples estudiados, aplicación de riego; tipo, momento de aplicación y dosis de antitranspirantes, tuvieron un efecto estadísticamente significativo sobre la vida en florero (días) con un 95.0 por ciento de nivel de confianza ( $\mathrm{p}$-valor $<0.05$ ).

Tabla 9. Vida en florero en la interacción aplicación de riego y momento de aplicación del antitranspirante

\begin{tabular}{|c|c|c|}
\hline \multicolumn{2}{|c|}{ Interacción } & \multirow{2}{*}{$\begin{array}{l}\text { Vida en florero } \\
\text { (días) }\end{array}$} \\
\hline Riego & Cosecha & \\
\hline \multirow{2}{*}{ Sin riego } & Precosecha & $14.2 \mathrm{a}$ \\
\hline & Poscosecha & $14.1 \mathrm{a}$ \\
\hline \multirow{2}{*}{ Con riego } & Precosecha & $16.2 \mathrm{~b}$ \\
\hline & Poscosecha & $17.4 \mathrm{~b}$ \\
\hline
\end{tabular}

En la primera interacción; riego y el momento de aplicación de los antitranspirantes, se observó una vida en florero significativamente mayor en las inflorescencias que recibieron riego y antitranspirante en pre y poscosecha, siendo de 16.2 días y 17.4 días respectivamente (Tabla 9). En la segunda interacción; tipo de antitranspirante y 
dosis del mismo, se encontró que las inflorescencias que recibieron el antitranspirante Vapor Gard $\AA$ a una dosis del 1 por ciento mostraron una vida en florero significativamente mayor (18.1) que los demás tratamientos; del mismo modo las inflorescencias a las que se aplicó Stress Relief $35 \circledR$ al 1 por ciento y Vapor Gard $\AA$ al 0.5 por ciento con longevidades florales de 15.9 y 16.1 respectivamente, mostraron diferencias significativas con las dosis de 0 por ciento de ambos antitranspirantes, 13.8 días (Tabla 10).

Tabla 10. Vida en florero en la interacción: tipo y dosis de antitranspirante

\begin{tabular}{ccc}
\hline \multicolumn{1}{c}{ Interacción } & & $\begin{array}{c}\text { Vida en florero } \\
\text { (días) }\end{array}$ \\
\hline Antitranspirante & Dosis & $13.8 \mathrm{a}$ \\
Stress Relief 35® & 0.0 & $14.6 \mathrm{ab}$ \\
& 0.5 & $15.9 \mathrm{~b}$ \\
\hline \multirow{2}{*}{ Vapor Gard $\mathbb{}$} & 1.0 & $13.8 \mathrm{a}$ \\
& 0.0 & $16.1 \mathrm{~b}$ \\
& 0.5 & $18.1 \mathrm{c}$ \\
\hline
\end{tabular}

En el factor simple: aplicación de riego, las inflorescencias de plantas que recibieron riego antes de ser cosechadas, tuvieron una vida en florero significativamente mayor (16.8 días) que aquellas que no recibieron riego antes de ser cosechadas, 14.1 días (Tabla 11).

En el factor simple: tipo de antitranspirante, Vapor Gard $\AA$ o Stress Relief 35®, las inflorescencias que fueron tratadas con Vapor Gard ${ }^{\circledR}$ tuvieron una vida en florero significativamente mayor (15.9 días) que las tratadas con Stress Relief ${ }^{\circledR}, 14.9$ días (Tabla 11).

Tabla 11. Vida en florero en los factores simples: aplicación de riego, tipo de antitranspirante, dosis y momento de cosecha.

\begin{tabular}{|c|c|c|c|c|c|c|c|c|}
\hline \multicolumn{2}{|c|}{ Riego } & \multicolumn{2}{|c|}{ Antitranspirante } & \multicolumn{3}{|c|}{ Dosis (\%) } & \multicolumn{2}{|c|}{ Cosecha } \\
\hline $\operatorname{Sin}$ & Con & & $\begin{array}{l}\text { Vapor } \\
\text { Gard }\end{array}$ & 0.0 & 0.5 & 1.0 & Pre & Post \\
\hline & & $14.9 \mathrm{a}$ & $15.9 \mathrm{~b}$ & & 5.6 & 17.10 & 15.2 & 5.7 \\
\hline
\end{tabular}

En el factor simple: dosis de aplicación de antitranspirante, las inflorescencias que recibieron la dosis más alta (1 por ciento) fueron las que tuvieron resultados significativamente superiores (17.1 días) a las demás concentraciones de ( 0.5 por ciento y 0 por ciento). Cabe resaltar, que aquellas inflorescencias que no recibieron antitranspirante ( 0 por ciento) también tuvieron una vida en florero significativamente menor (13.8 días) que aquellas tratadas con la dosis de 0.5 por ciento de antitranspirante, 15.6 días (Tabla 11).

En el factor momento de aplicación del antitranspirante, las inflorescencias tratadas con antitranspirantes después de ser cosechadas, tuvieron una vida en florero significativamente mayor (15.7 días) que aquellas inflorescencias tratadas antes de ser cosechadas, 15.2 días (Tabla 11).
En Heliconia bihai, al igual que el resto de las heliconias, la capacidad de tomar agua declina considerablemente luego de la cosecha (Ka-ipoet al., 1989). Esto es debido a que sus inflorescencias son afectadas en gran medida por el bloqueo xilemático. De ahí la importancia de que las inflorescencias de esta especie se deshidraten lo menos posible en poscosecha y más aún que las inflorescencias se encuentren en el mejor estado de turgencia antes del corte de las inflorescencias.Además es importante disminuir la pérdida de agua por transpiración, para prolongar la vida poscosecha de las inflorescencias de las heliconias. La aplicación correcta de antitranspirantes, como se observó en el experimento realizado, reduce la pérdida peso (Criley y Paull, 1993).

En las heliconias, la calidad poscosecha que determina la aceptación del producto, está ligada al daño observado en la superficie de sus brácteas cincinales. En el estudio realizado, el daño observado en la superficie de las brácteas cincinales en inflorescencias de Heliconia bihai fue concordante con el surgimiento de manchas oscuras en el tejido de las brácteas, así como un amarillamiento y desecamiento de la punta de las brácteas y el peciolo de las hojas, que posiblemente fue ocasionado por una elevada tasa respiratoria. Esto concuerda con los daños observados por Whittaker (1993) para otras especies del género Heliconia y por Andrade (2008) para Heliconia bihai. Por esto, aquellos tratamientos en los que se logró mejorar el estado hídrico de las inflorescencias (mediante el riego 12 horas antes de la cosecha) y se redujo la pérdida de agua por transpiración (mediante uso de antitranspirantes) mostraron un resultado positivo en cuanto a la reducción del porcentaje de daño observado en la superficie de las brácteas cincinales de las inflorescencias de Heliconia bihai.

Muchos factores de precosecha determinan la composición y calidad poscosecha de los productos hortícolas. En ese sentido la calidad comienza a determinarse mucho antes de la cosecha y es importante conocer la manera en que estos factores afectan el comportamiento poscosecha. Uno de los factores que condicionan la calidad en el momento de la cosecha y poscosecha es el contenido y momento de aplicación del agua de riego. El sistema y momento del aporte hídrico también afectan a la calidad y la aparición de fisiopatías y podredumbres durante la poscosecha. (Romojaro et al., 2003).

La vida poscosecha de la mayoría de las flores de corte depende de dos factores que son el control hormonal de la senescencia (generalmente el nivel de producción de etileno) y las relaciones hídricas. En el caso de las inflorescencias de heliconias, la producción de etileno no define su senescencia, en cambio el decrecimiento en el consumo de agua y la transpiración sí afecta su vida en florero (Van Doorn et al., 1999). Según Ka-ipo et al. (1989), esta deficiencia en el consumo de agua después de la cosecha se debe al bloqueo xilemático en los tallos de las inflorescencias de las heliconias; el efecto de este bloqueo fue suprimido por Andrade (2008) quien prolongó 
la vida poscosecha de inflorescencias de Heliconia bihai mediante el corte de los primeros centímetros de la base del tallo de las inflorescencias cada 24 horas; sin embargo no resulta un método práctico ni para comerciantes ni para el consumidor final.

Castro et al (2006) menciona que las heliconias que presentan una longevidad menor o igual a 7 días son clasificadas como de vida corta, de 8 a 14 días media y aquellas que se mantienen con calidad satisfactoria mayor a 14 días poseen longevidad alta. En el presente estudio se encontró una vida en florero mínima de 12.3 días y máxima de 20.7 días por lo que se ha podido llevar a las inflorescencias de Heliconia bihai de una longevidad media hasta una longevidad alta mediante el riego previo a la cosecha y la aplicación de antitranspirantes.

Andrade (2008) reportó una vida poscosecha de Heliconia bihai de 7 a 12 días y menciona que el fin de la misma se presenta cuando la inflorescencia presenta algún tipo de daño en el 50 por ciento o más de su superficie; sin embargo en el presente estudio se encontró una vida en florero mayor en todos los tratamientos. Una diferencia de longevidades florales tan marcada como la encontrada entre el presente estudio y el realizado Andrade (2008) podría deberse al estado de desarrollo de las inflorescencias que fue distinto, correspondiendo un estado de desarrollo mayor y una vida en florero menor en el estudio de Andrade (2008). Esto corrobora lo mencionado por Jaroenkit y Paull (2003) que la vida en florero de las heliconias varía dependiendo del cultivar, estado de desarrollo de las flores al momento de cosecha y momento del día en el que se cosechó la inflorescencia.

\section{Conclusiones}

Aquellas inflorescencias de plantas de Heliconia bihai que recibieron riego 12 horas antes de la cosecha y la aplicación del antitranspirante Vapor Gard ${ }^{\circledR}$ (en cualquiera de sus dosis), 24 horas antes de la cosecha o inmediatamente después de la misma, mostraron una pérdida de peso significativamente menor que los demás tratamientos.

Aquellas inflorescencias de plantas de Heliconia bihai que recibieron riego 12 horas antes de la cosecha y la aplicación del antitranspirante Vapor Gard ${ }^{\circledR}$ a una dosis del 1 por ciento mostraron un daño observado significativamente menor.

Las inflorescencias de Heliconia bihai con aplicación del

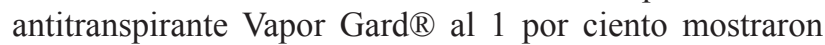
significativamente menor pérdida de peso, menor daño observado, mayor límite de comercialización y mayor vida en florero.

Las inflorescencias de Heliconia bihai que recibieron riego 12 horas antes de la cosecha y a las que se aplicó algún antitranspirante 24 horas antes de la cosecha o inmediatamente después de la misma, mostraron significativamente mayor vida en florero.

La aplicación de riego a plantas de Heliconia bihai 12 horas antes de la cosecha tuvo un efecto positivo en la disminución del porcentaje de pérdida de peso y daño observado; y en la prolongación del límite de comercialización y la vida en florero de sus inflorescencias.

La aplicación del antitranspirante Vapor Gard $\AA$ tuvo un mayor efecto en la disminución del porcentaje de pérdida de peso y daño observado; y en la prolongación del límite de comercialización y la vida en florero de las inflorescencias de Heliconia bihai que la aplicación del antitranspirante Stress Relief 35®.

La aplicación de cualquiera de los antitranspirantes utilizados a dosis del 1 por ciento tuvo efecto en la disminución del porcentaje de pérdida de peso y daño observado; y en la prolongación del límite de comercialización y la vida en florero de las inflorescencias de Heliconia bihai.

La aplicación de los antitranspirantes estudiados inmediatamente después de la cosecha tuvo mayor efecto en la disminución del porcentaje de pérdida de peso y daño observado; y en la prolongación del límite de comercialización y la vida en florero de las inflorescencias de Heliconia bihai que la aplicación del antitranspirante 24 horas antes de la cosecha.

\section{Literatura citada}

Andrade, A. 2008. Manejo Pós-colheita de hastes florais de Heliconia bihai. Tesis de Doctorado. Universidad Federal de Vicosa. Minas Gerais. Brasil. 181 p.

Berry, F. and Kress, W. 1991. Heliconia: an identification guide. US. Smithsonian Institution Press. 333 p.

Castro, C. E. F.; May, A. y Gonçalves, C. 2006. Espécies de helicônia como flores de corte. Revista Brasileira de Horticultura Ornamental, 12:.87-96.

Criley, R. and Paull, R. 1993. Review: Postharvest handling of bold tropical cut flowers Anthurium, Alpinia purpurata, Heliconia, and Strelitzia. Acta Horticulturae 37: $1-21$.

Van Doorn, W. V. 1999. Water relations of cut flowers II: Some species of tropical provenance. Acta Horticulturae 482: 65-69.

Jaroenkit, T. and Paull, R. 2003. Postharvest handling of heliconia, red ginger, and bird-of-paradise. Hort Technology 13(2): 259-266.

Ka-Ipo, R.; Sakai, W.; Furutani, S. and Collins, M. 1989. Effect of postharvest treatment with antitranspirants on the shelf-life of Heliconia psittacorum cv. 'Parakeet' cut flowers. Bulletin of the Heliconia Society International 4: 13-14.

Rocha, N. 2010. Diseño de una metodología de diagnóstico para empresas productoras de flores tropicales y follajes en el eje cafetero. Tesis de Maestría en Ciencias Agrarias. Universidad Nacional de Colombia.125 p.

Romojaro, F.; Martinez, M. y Petrel, M. 2003. Factores precosecha determinantes de la calidad y conservación en poscosecha de productos agrícolas. ES. Ministerio 
de Ciencia y Tecnología. p. 91 - 96.

Torres, N. 2003. Análisis de desarrollo empresarial para la producción de heliconias y follajes tropicales en las veredas Canaán y la Palmera ubicadas en el municipio de Salento. Bogotá, CO. Instituto Alexander von Humboldt. 83 p.

Whitakker, J. 1993. Postharvest handling procedure for Jamaican grown cut flowers. Tesis de Maestría. Universidad de Gainsville. Florida. Estados Unidos. 98 p. 